\title{
Necessidades de aprendizagem de familiares de crianças e adolescentes em tratamento com quimioterápicos antineoplásicos orais
}

\author{
Learning needs of relatives of children and teenagers under oral antineoplastic chemotherapy treatment \\ Necesidades de aprendizaje de los familiares de niños y adolescentes en tratamiento con quimioterapia \\ antineoplástica oral
}

Gabriele Alvernaz Silva Franco ${ }^{1}$ (1)

Liliane Faria da Silva² (1)

Flavio Luiz Seixas ${ }^{2}$ (1)

Fernanda Garcia Bezerra Góes ${ }^{3}$ (D)

Ana Carolina Alves Vollu² (D)

Eduardo Charpinel Lagoeiro² (1)

1. Instituto Nacional do Câncer. Rio de Janeiro,

RJ, Brasil.

2. Universidade Federal Fluminense. Niterói, RJ, Brasil.

3. Universidade Federal Fluminense. Rio das Ostras, RJ, Brasil.

\section{RESUMO}

Objetivo: descrever as necessidades de aprendizagem de familiares de crianças e adolescentes com câncer quanto ao tratamento com quimioterápicos antineoplásicos orais. Método: pesquisa qualitativa descritiva desenvolvida em um hospital federal do Rio de Janeiro, Brasil. Os dados foram coletados nos meses de julho a setembro de 2020 a partir de entrevistas semiestruturadas com vinte e três familiares de crianças e adolescentes com câncer em quimioterapia antineoplásica oral. Os dados foram processados no software Interface de R pour Analyses Multidimensionnelles de Textes et de Questionnaires pela Classificação Hierárquica Descendente. Resultados: dentre os temas que demandam aprendizagem pelos familiares estão administração oral, armazenamento e manipulação dos quimioterápicos orais, além dos efeitos adversos e emergências que demandam atendimento hospitalar. Conclusão e implicações para a prática: no tratamento com quimioterápicos orais, as necessidades de aprendizagem dos familiares de crianças e adolescentes precisam ser problematizadas em práticas educativas dialógicas para, assim, favorecer a segurança, a adesão e a eficácia do tratamento.

Palavras-chave: Administração Oral; Adolescente; Antineoplásicos; Criança; Família.

\section{ABSTRACT}

Objective: to describe the learning needs of family members of children and adolescents with cancer regarding treatment with oral antineoplastic chemotherapies. Method: a descriptive qualitative research developed in a federal hospital in Rio de Janeiro, Brazil. Data were collected in the months from July to September 2020 from semi-structured interviews with twenty-three family members of children and adolescents with cancer undergoing oral antineoplastic chemotherapy. Data was processed in the software Interface de R pour Analyses Multidimensionnelles de Textes et de Questionnaires by the Descending Hierarchical Classification. Results: among the themes that demand learning by the family members are oral administration, storage and handling of oral antineoplastic drugs, as well as adverse effects and emergencies that require hospital care. Conclusion and implications for practice: in oral antineoplastic treatment, the learning needs of family members of children and adolescents need to be problematized in dialogic educational practices in order to favor the safety, adherence, and efficacy of the treatment.

Keywords: Oral Administration; Adolescent; Antineoplastic Agents; Children; Family.

\section{RESUMEN}

Objetivo: describir las necesidades de aprendizaje de familiares de niños y adolescentes con cáncer en cuanto al tratamiento con quimioterápicos antineoplásicos orales. Método: investigación cualitativa descriptiva desarrollada en un hospital federal de Río de Janeiro, Brasil. Los datos fueron recogidos en los meses de julio a septiembre de 2020 a partir de entrevistas semiestructuradas con veintitrés familiares de niños y adolescentes con cáncer en quimioterapia antineoplásica oral. Los datos fueron procesados en el software Interface de R pour Analyses Multidimensionnelles de Textes et de Questionnaires por la Clasificación Jerárquica Descendente. Resultados: entre los temas que demandan aprendizaje por los familiares están administración oral, almacenamiento y manipulación de los quimioterápicos orales, además de los efectos adversos y emergencias que demandan atención hospitalaria. Conclusión e implicaciones para la práctica: en el tratamiento con quimioterápicos orales, las necesidades de aprendizaje de los familiares de niños y adolescentes necesitan ser problematizadas en prácticas educativas dialógicas para, así, favorecer la seguridad, la adhesión y la eficacia del tratamiento.

Palabras clave: Administración oral; Adolescente; Antineoplásicos; Niños; Familia.
Autor correspondente:

Gabriele Alvernaz Silva Franco.

E-mail: gabrielealvernaz@yahoo.com.br

Recebido em 06/07/2021.

Aprovado em 15/12/2021.

DOI:https://doi.org/10.1590/2177-9465-EAN-2021-0246 


\section{INTRODUÇÃO}

A incidência do câncer infantojuvenil tem aumentado nos últimos anos no Brasil e no mundo ${ }^{1}$ e este estudo aborda as drogas quimioterápicas que, no tratamento de crianças e adolescentes com câncer, contribuem, de um modo relevante, para as taxas de sobrevida, já que os tumores pediátricos têm alto índice proliferativo e são, na sua maioria, quimiossenssíveis. ${ }^{1,2}$

Durante muitos anos, o tratamento farmacológico do câncer utilizou, exclusivamente, a terapia antineoplásica intravenosa e os serviços de saúde foram estruturados com base nesse modelo de tratamento. Todavia, nas últimas décadas, foi observado um aumento exponencial no desenvolvimento e no uso de drogas antineoplásicas orais com efeitos tóxicos menos agressivos que, além de bem toleráveis, também são de fácil manejo. ${ }^{3}$

Estudos apontaram as vantagens e as desvantagens dessa terapêutica. As vantagens da administração oral referem-se ao fato de ela ser mais simples, econômica, menos tóxica e não invasiva, eliminando a necessidade de uso de cateter venoso que desencadeia dor, medo e estresse nas crianças e nos adolescentes. Além disso, poder ser administrada no ambiente domiciliar, pela família, o que implica mais tempo de convivência com os familiares e menos tempo em hospitais, promovendo, assim, maior qualidade de vida desse público. ${ }^{3,4}$

Já as desvantagens são as variações na absorção da dose terapêutica; o risco de acidentes com superdosagem; a necessidade de autocuidado eficiente e a dificuldade de manejo dos efeitos colaterais. Esses riscos podem potencializar as chances de não adesão à terapêutica oral e gerar efeitos prejudiciais. Assim, esse tipo de tratamento pode transformar-se em um enorme problema se os familiares não forem, adequadamente, orientados e preparados para efetuá-lo. 3,4

Compreender como armazenar, preparar, manusear, administrar e descartar, adequadamente, os antineoplásicos orais é necessário para reduzir o risco de exposição e garantir que a criança e o adolescente recebam a dose terapêutica adequada. Estudo de revisão sistemática da literatura avaliou a gestão de pacientes e cuidadores sobre os antineoplásicos orais, identificando a necessidade de desenvolver estratégias de educação que abordem a administração neste cenário, pois muitos não conseguem realizar tarefas essenciais de segurança, como lavar as mãos antes e após a administração, a eliminação dos resíduos e dúvidas quanto à manipulação. ${ }^{5}$

Outro estudo realizado com 64 cuidadores de pacientes pediátricos com leucemia linfoblástica aguda destacou que o gerenciamento inadequado de agentes antineoplásicos representa um risco de exposição a substâncias perigosas para as pessoas envolvidas, inclusive por meio de superfícies domésticas e meio ambiente. Acrescentou que os erros no gerenciamento desses medicamentos surgiram, principalmente, devido à falta de conhecimento dos cuidadores sobre o manuseio dos antineoplásicos orais. ${ }^{6}$

Assim sendo, as famílias de crianças e adolescentes que fazem uso de quimioterápicos na apresentação oral devem receber acompanhamento especial e orientações de uma equipe multidisciplinar. O preparo adequado torna-se fundamental nesse processo, guiando o uso correto das drogas, acompanhando reações adversas e interações, a fim de diminuir os riscos de erros e a descontinuidade do tratamento. Nesse contexto, as orientações, por parte dos enfermeiros, tornam-se cruciais, pois favorecem a participação efetiva da família nos cuidados. ${ }^{7}$

É papel do enfermeiro, durante a consulta de Enfermagem, dialogar com o familiar sobre as recomendações no acompanhamento do paciente, incluindo orientações sobre os quimioterápicos e outros medicamentos associados ao tratamento e dicas quanto às possíveis reações adversas. ${ }^{8}$ Enfatiza-se, portanto, a atribuição do enfermeiro como educador, pois o propósito final é contribuir para o sucesso do tratamento e a reintegração da criança e do adolescente à sua rotina de vida, de modo a estimular que estes e seus familiares deixem o papel de sujeitos passivos e passem a relacionar-se com a equipe, revelando suas necessidades de aprendizagem, dúvidas, anseios e sentimentos durante o tratamento. ${ }^{9,10}$

Assim, este estudo apoia-se nas concepções teóricas da educação problematizadora de Paulo Freire ${ }^{11}$ que versa sobre a prática de liberdade que rompe com a verticalidade da prática bancária, e propõe, por meio da dialogicidade, uma relação transversal entre os sujeitos. Ao partir desse prisma, buscam-se o diálogo e a reflexão crítica da realidade para o desenvolvimento de qualquer ação educativa. Assim, mais do que a aquisição de conhecimentos provenientes de uma translação de saberes, almeja-se que o conhecimento seja construído, vivenciado e harmonizado de forma coletiva e participativa. ${ }^{12}$

Portanto, antes da implementação de práticas educativas em saúde, especialmente em se tratando de crianças e adolescentes dependentes de cuidados domiciliares, é preciso ouvir os familiares, conhecer suas necessidades de aprendizagem, dúvidas e medos para, assim, pensar em estratégias que visem ao melhor para a família, a criança e o adolescente. ${ }^{13}$ Contudo, evidências científicas acerca das reais necessidades de aprendizagem de familiares frente ao tratamento com quimioterápicos na apresentação oral entre a população infantojuvenil, que possam alicerçar a construção de práticas educativas pelos profissionais de saúde atuantes nessa área, incluindo os enfermeiros, ainda são escassas. Assim, o objetivo da pesquisa foi descrever as necessidades de aprendizagem de familiares de crianças e adolescentes com câncer quanto ao tratamento com quimioterápicos antineoplásicos orais.

\section{MÉTODO}

Pesquisa descritiva, de abordagem qualitativa, ${ }^{14}$ na qual foram adotados os critérios definidos pelo Consolidated Criteria for Reporting Qualitative Research (COREQ). ${ }^{15}$ A pesquisa foi realizada em um hospital federal localizado no município do Rio de Janeiro. Este é um órgão auxiliar do Ministério da Saúde no desenvolvimento e na coordenação das ações integradas para a prevenção e o controle do câncer no Brasil. ${ }^{1}$

O hospital possui duas centrais de quimioterapia: uma destinada ao atendimento do público adulto e outra, cenário 
do estudo, destinada ao atendimento do público infantojuvenil, cujo fluxo de atendimento médio é de 23 crianças/adolescentes por dia. Este setor funciona das sete às 19 horas, em todos os dias da semana, incluindo feriados. Para o atendimento, conta com duas enfermeiras plantonistas e uma diarista. A enfermeira desse setor é a responsável por administrar o quimioterápico dos pacientes internados na pediatria e hematologia infantil e no Centro de Terapia Intensiva (CTI) infantil, que atendem pacientes de até 19 anos de idade.

Participaram da pesquisa 23 familiares de crianças/adolescentes em tratamento com quimioterápico antineoplásico oral. Os critérios de inclusão foram: ser cuidador da criança/adolescente; ter experiência prévia de mais de um mês administrando o quimioterápico oral, tanto no ambiente ambulatorial quanto no domiciliar e ser maior de 18 anos. Foram excluídos os familiares de crianças e adolescentes que estavam hospitalizados no período da coleta de dados.

O recrutamento dos participantes foi realizado, pessoalmente, pela enfermeira mestranda, primeira autora. No período de coleta de dados, estavam registrados no serviço 45 crianças/ adolescentes em tratamento com quimioterápicos orais. Foram abordados 24 familiares, entretanto, um recusou-se a participar, relatando não se sentir confortável em ter suas falas gravadas. A delimitação do quantitativo de participantes ocorreu no decorrer do trabalho de campo por meio da saturação teórica dos dados identificada na organização dos depoimentos. ${ }^{16}$

Efetuou-se a coleta de dados nos meses de julho a setembro de 2020, mediante entrevista semiestruturada, utilizando-se um roteiro com perguntas fechadas e abertas. As perguntas fechadas correspondiam à caracterização dos participantes e as abertas, ao atendimento do objetivo da pesquisa. Desta maneira, foram realizadas as seguintes perguntas: "De que modo você cuida da criança/adolescente em tratamento com quimioterápicos orais? Quais são os cuidados que você tem com quimioterápicos orais? Quais são as suas dúvidas?".

As entrevistas foram conduzidas pela primeira autora inserida no cenário do estudo, tiveram duração média de vinte minutos e foram realizadas no consultório de Enfermagem da quimioterapia infantil, que é um local reservado. Foram gravadas com o auxílio de um gravador de voz para registro integral das falas dos participantes e, posteriormente, transcritas na íntegra.

Os dados foram processados por meio do software Interface de Rpour Analyses Multidimensionnelles de Textes et de Questionneires (IRAMUTEQ), que se configura como um método informatizado para processamento de textos (corpus), que perscruta a estrutura e a organização dos enunciados, exprimindo as associações entre os mundos lexicais. $O$ uso desse software viabilizou a codificação, organização e separação das informações, promovendo maior rigor metodológico. ${ }^{17}$ Desse modo, a análise dos dados textuais aconteceu em três etapas: 1) preparação e codificação do corpus textual com a descrição do material proveniente das entrevistas;2) processamento dos dados textuais no software e 3) interpretação dos dados obtidos pelos pesquisadores..$^{18}$

Na etapa de processamento, utilizou-se um dos cinco métodos de análise disponíveis, o método da Classificação Hierárquica
Descendente (CHD), visto que essa interface propicia, segundo o corpus original, a recuperação dos segmentos de texto e a correlação entre cada um por intermédio do grupamento das palavras estatisticamente significativas em classes. ${ }^{19,20}$

Para tal, o software realiza uma prova estatística profusamente conhecida em estudos de abordagem quantitativa, o qui-quadrado de Pearson, em que quanto maior, mais provável é a hipótese de dependência entre a palavra ativa e a classe. ${ }^{18}$ Assim, as palavras que apresentam $x 2$ igual ou superior a 3,84 $(p<0,05)$ apresentam associação na classe, com destaque para as com $\mathrm{p}<0,0001$, com fortíssima associação. ${ }^{18} \mathrm{Em}$ seguida, a etapa de interpretação das classes, dos segmentos de textos e das palavras associadas a elas ocorreu à luz do referencial teórico do estudo ${ }^{11}$ e de outras evidências científicas sobre a temática.

A pesquisa foi aprovada pelo Comitê de Ética em Pesquisa da instituição proponente (CAAE: 30134320.2.0000.5243; número do Parecer 4.012.929) e da instituição coparticipante onde a pesquisa foi realizada (CAAE: 30134320.2.3001.5274; número de Parecer 4.074.187, em junho de 2020) e os dados foram produzidos mediante a assinatura do Termo de Consentimento Livre Esclarecido (TCLE).

Os participantes tiveram identidade preservada e foram identificados por meio de códigos referentes ao número de entrevista, sendo F1 0 primeiro familiar entrevistado, F2 o segundo familiar entrevistado e, assim, sucessivamente, a fim de ser mantido o anonimato. Vale destacar que a pesquisadora assumiu o compromisso de zelar pela integridade dos participantes da pesquisa durante a coleta de dados com os familiares e obedeceu a todos os cuidados necessários frente ao risco de transmissão do novo Coronavírus de acordo com as orientações gerais para a condução de protocolos de pesquisa no cenário da SARS-CoV-2 (COVID-19). ${ }^{21}$

\section{RESULTADOS}

Participaram 23 familiares (100\%) de crianças e adolescentes em uso de quimioterápicos antineoplásicos orais, dentre estes, dezesseis mães (69,6\%), quatro pais (17,5\%), dois irmãos $(8,6 \%$ ) e uma avó (4,3\%). A maior parte dos familiares estava na faixa etária dos 40 anos (52,2\%), com Ensino Médio completo $(52,17 \%)$ e $87 \%$ das famílias possuíam outros filhos. Quanto às características das crianças/adolescentes, a idade mais prevalente foi de dez anos (39,4\%) e, em relação ao diagnóstico, encontraram-se doze em tratamento para doenças oncohematológicas, sendo que o tipo mais predominante foi a leucemia, enquanto onze estavam em tratamento para tumores sólidos, dentre os quais $54 \%$ eram de tumores do sistema nervoso central. Os quimioterápicos orais mais utilizados foram a Mercaptopurina (47\%) e o Metotrexato $(26 \%)$, indicados para o tratamento de doenças hematológicas como as leucemias, linfomas e histiocitose. A Temozolamida (13\%) também obteve destaque, sendo utilizada para o tratamento de tumores do sistema nervoso central.

A CHD reteve 256 segmentos de texto, dos 23 textos processados, classificando-os em cinco classes estáveis, com índice de aproveitamento de 84,77\%, conforme o dendrograma (Figura 1), que expõe as relações entre essas classes e o percentual de cada uma quanto ao total do corpus analisado. 


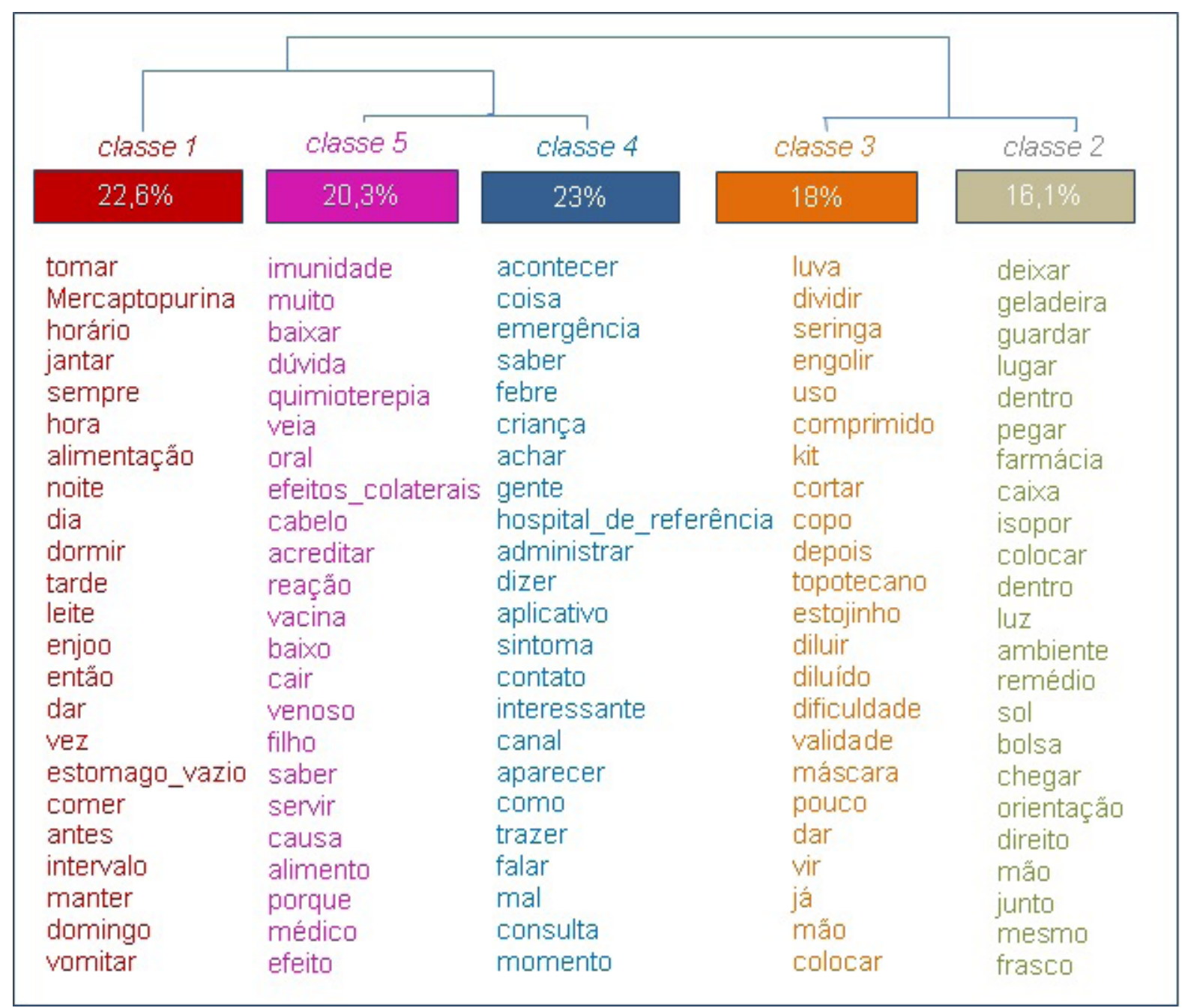

Figura 1. Dendrograma da CHD dos segmentos de texto relacionados às necessidades de aprendizagem de familiares de crianças e adolescentes com câncer quanto ao tratamento com quimioterápicos antineoplásicos orais. Niterói, RJ, Brasil, 2020. Fonte: fornecida pelo software IRAMUTEQ.

As cinco classes foram estáveis, ou seja, compostas por segmentos de texto com vocábulos semelhantes, constituindo, assim, distintos mundos lexicais de acordo com parâmetros léxicos em comum e vocabulário distinto das outras classes. A leitura da relação entre as classes realizada no dendrograma é feita de cima para baixo. Assim, o primeiro subcorpus sofre duas divisões, nas classes 3 e 2. Por meio do dendrograma, foi possível contemplar as palavras que alcançaram maior porcentagem quanto à frequência média entre si e diferente entre elas. ${ }^{17}$

Após a leitura exaustiva dos segmentos de texto de cada classe, foi possível alcançar os objetivos da pesquisa, denominando as cinco classes oferecidas pelo software de acordo com os temas emergentes da interpretação dos achados. As classes serão apresentadas a seguir com base nas principais palavras associadas em cada classe, porém, relacionando-as aos segmentos de texto dos quais elas emergiram, conferindo contexto ao que foi dito pelos entrevistados à luz do referencial teórico do estudo.

\section{Classe 1 - Administração dos quimioterápicos orais}

Nesta classe, os familiares descreveram como a administração do quimioterápico oral é feita, revelando, assim, distintas necessidades de aprendizagem sobre esse tópico. Assim, as principais palavras associadas foram: tomar; mercaptopurina; horário; jantar; sempre; hora; alimentação. As descrições a seguir evidenciam alguns aspectos que são considerados primordiais na realização dos cuidados por esses familiares, tais como seguir os horários recomendados e a necessidade de modificação da rotina alimentar da criança/adolescente.

Eu cuido cumprindo os horários de tomar o quimioterápico. Procuro sempre manter o horário certo do remédio e, com a alimentação, só dou o que pode comer. (F5)

Nós mudamos a alimentação: durante o dia, ele tem uma alimentação balanceada, frutas e sucos. Então, ele 
almoça e, depois, a gente dá o remédio; é uma vez ao dia, sempre às 12 horas em ponto. (F19)

Outros familiares relataram algumas necessidades de aprendizagem relacionadas ao horário da quimioterapia como não saber se o quimioterápico pode ser administrado com alimentação e quanto ao fato de manusear o comprimido com a mão ou não. Há ainda, nos relatos, uma divergência no modo como é administrado o quimioterápico "mercaptopurina": o familiar 22 relatou não oferecer leite, enquanto o familiar 21 oferece com leite depois de um tempo da administração do medicamento.

Não faço distinção se ele jantou ou não, ninguém me passou nada assim, então, eu mantenho o horário das 21 horas. Geralmente, eu pego o comprimido na mão e coloco na mão dele e ele toma. (F17)

Damos a Mercaptopurina sempre à noite porque não podia dar com leite, então, damos junto com a primeira ou com a última colherada da janta. (F22)

A Mercaptorpurina é de segunda a segunda sem intervalo, às 22 horas da noite. Primeiro, ele come alguma coisa e, depois, toma o remédio; espero passar um pouco e dou um copo de leite para ele não ficar com o estômago vazio. (F21)

Nas experiências, destaca-se que os familiares das crianças/ adolescentes em tratamento para as doenças oncohematológicas possuem um esquema de protocolo diferenciado, com mais de um quimioterápico oral $(30,4 \%)$, e que eles são prescritos de formas diferentes ao longo da semana, podendo ser um fator de confusão na hora da administração, o que demanda a necessidade de reforço nas orientações por parte dos profissionais.

Todos os dias tem um comprimido: a Mercaptopurina ela toma todos os dias e o MTX (metotrexato) ela toma seis comprimidos uma vez na semana, que é no domingo, $e$, assim, a gente tem que ficar observando. (F12)

Ela toma a Mercaptopurina às 18 horas da tarde, um comprimido e meio, de segunda a sábado e, no domingo, ela toma dois comprimidos. OMTX (metotrexato) ela faz 11 comprimidos só no sábado. Eu já esqueci algumas vezes de dar o quimioterápico. (F23)

O familiar F16 relatou que a sua principal dúvida é em relação à quantidade de comprimidos, pois a criança toma uma quantidade de comprimidos durante a semana e, no final de semana, é uma quantidade diferente do mesmo quimioterápico.

A dúvida que eu tenho é em relação à quantidade de comprimido porque tem uma dosagem certa, se ela vai tomar inteiro ou a metade. (F16)
Os familiares F20 e F22 relataram como eles procediam no caso de vômitos antes ou após a administração do quimioterápico, inclusive mostrando que a decisão de não oferecer novo medicamento era pautada na própria observação e não em orientação profissional.

Teve dias dele tomar o quimioterápico e vomitar em seguida; nós não dávamos outro não, até porque ele estava vomitando constantemente. Então, não ia adiantar dar outro, seria desperdiçar o remédio. (F20)

Nós não demos o quimioterápico algumas vezes sim, não foi por esquecimento, isso foi nos dias em que ele acordava vomitando, a gente não dava a Mercaptopurina. Isso não foi uma orientação médica, nós que seguimos dessa forma. (F22)

\section{Classe 2 - Armazenamento dos quimioterápicos orais}

Nesta classe, as palavras mais associadas às necessidades de aprendizagem dos familiares foram: deixar; geladeira guardar; lugar; dentro. Elas remetem à forma como os familiares realizam o armazenamento da medicação no domicílio, segundo suas próprias experiências. Nesse sentido, alguns familiares destacaram que estes ficam guardados no mesmo local, em caixa, junto com todos os outros remédios em uso pela criança/ adolescente, sem que fossem tomados cuidados diferenciados por tratar-se de quimioterápicos antineoplásicos.

Guardo o quimioterápico dentro de um saco próprio junto com todos os outros remédios dela. (F6)

Eu guardo os quimioterápicos numa caixa fechada junto com todos os outros remédios dela. (F23)

A familiar F14 relatou que, ao armazenar o quimioterápico, separa os comprimidos que estão inteiros dos que já estão cortados. Assim, reduz o contato com os cortados, ou seja, com aqueles que foram manuseados pelo familiar no domicílio.

Eu guardo os remédios em dois vidros: um para os comprimidos inteiros e o outro vidro eu deixo para os comprimidos cortados; eu deixo separado para não ficar movimentando muito os comprimidos cortados. (F14)

Com relação à temperatura, um familiar referiu deixar o medicamento em temperatura ambiente, destacando incerteza quanto à conduta, pois relatou não saber se havia lido, corretamente, a orientação. Já outro familiar relatou manter em geladeira, embora afirme não saber em qual parte da geladeira deve deixar.

Não sei se eu li certo. Mas, eu guardo o remédio em temperatura ambiente, não deixo no sol, num lugar seco, não coloco na geladeira. (F17) 
A minha dúvida é muito a questão do armazenamento; eu chego em casa com o remédio no isopor e coloco na geladeira, eu não sei qual é a parte da geladeira que eu tenho que deixar. (F8)

Os relatos evidenciaram que os familiares realizam o armazenamento dos quimioterápicos orais de distintas formas. Alguns são acondicionados em geladeira e em outros locais, como cozinha, quarto, sacolas e bolsas. Essa não uniformidade também se apresenta com uma necessidade de aprendizagem em relação ao armazenamento, pois as decisões ainda estão pautadas em percepções, observações e experiências adquiridas na execução do cuidado e não em práticas educativas contextualizadas e problematizadoras.

\section{Classe 3 - Manipulação dos quimioterápicos orais}

As palavras que obtiveram maior frequência de associação nesta classe foram: luva; dividir; seringa; engolir; uso; comprimido. Os segmentos de texto e as palavras que caracterizaram este tema traduzem como os familiares manuseiam e manipulam os quimioterápicos orais no domicílio, bem como indicam suas necessidades de aprendizagem.

Alguns familiares pegavam o quimioterápico oral e iam ao serviço de farmácia quimioterápica para que fossem manuseados e, assim, recebiam um kit fechado, contendo as seringas com o quimioterápico diluído, luvas, copos e sacola para colocar as seringas vazias para o descarte no hospital. Neste sentido, seguiam os cuidados que foram orientados no manuseio do medicamento.

Junto com as seringas, a farmácia me deu um kit. Vêm a luva, uns copinhos para diluir e a seringas já prontas com o remédio. (F7)

Eu só uso as luvas que vêm no kit para dar o Topotecano para minha filha e vou juntando as seringas na sacola $e$ desprezo no hospital. A Ciclofosfamida, eu dou normal com a mão e jogo a cartela no lixo de casa. (F15)

Por outro lado, outros familiares relataram que não encaminham os quimioterápicos para diluição na farmácia, mas que realizam essa manipulação (cortar, dividir e diluir) do quimioterápico oral no próprio domicílio. Esses achados reforçam a necessidade de serem orientados para maior segurança no manuseio desses medicamentos.

Eu tenho um estojinho de acrílico para cortar o comprimido. Eu divido ele no meio e, depois, corto no meio de novo, não uso luva para dividir o comprimido. (F14)

Nós dividimos o remédio, é só meio comprimido a dosagem dele. Então, a gente sempre corta. E a gente sempre apoia na toalha de mesa mesmo, limpa. (F22)

Eu coloco um pouco de água num copinho e deixo o comprimido derretendo e, depois, eu dou para ele engolir.
Porque ele tem dificuldade para engolir todos os tipos de comprimido. (F18)

Além disso, o familiar F14 relatou que não recebeu orientações quanto à manipulação e à diluição do quimioterápico.

Como ela é muito pequena, não consegue engolir o comprimido. Eu dissolvo ele em um copinho. Ninguém me orientou como eu deveria diluir o comprimido, eu comprei uma mamadeira pequena. (F14)

As falas mostraram que, quando os familiares não são nem orientados de modo contextualizado, nem direcionados a adquirir o kit com os materiais necessários para o adequado manuseio do quimioterápico oral no domicílio, eles realizam o cuidado do mesmo modo que fazem com outros medicamentos não quimioterápicos.

\section{Classes 4 - Necessidade de orientação dos familiares}

Os familiares relataram os principais assuntos que gostariam que fossem abordados nas orientações para o cuidado domiciliar. As palavras que obtiveram maior frequência de associação foram: acontecer; coisa; emergência; saber; febre. Nas entrevistas a seguir, os familiares sinalizaram que gostariam que fossem orientados quanto aos efeitos adversos.

Seria bem bacana saber sobre o que pode acontecer, qual o efeito que o remédio faz na criança. (F23)

Gostaria de saber sobre a reação porque, se você sabe qual a reação que dá, você administra bem, se você não sabe que pode dar uma febre, uma tonteira, a criança pode ficarneutropênica e você não sabe e, às vezes, é por causa do quimioterápico oral, e isso é muito importante: a orientação. (F9)

Além dos efeitos colaterais, os familiares gostariam de ter informação quanto às circunstâncias que exigem levar a criança/ adolescente à emergência do hospital de referência.

Se a criança pode ter algum tipo de reação, alguma coisa que possa preparar quem está com a criança para poder vir para o hospital de referência. (F21)

Alguns familiares solicitaram orientações com relação à manipulação dos quimioterápicos: se podem ou não colocar as mãos; como dividir os remédios; o lugar correto para o armazenamento e descarte.

Como manipular? Porque eu mesmo pego com a mão, corto o remédio, dou para ela e ela toma. Nunca fui informada em como dividir o remédio. (F23) 
Como a gente deve manipular o quimioterápico, não colocar a mão, como a gente guarda e qual o lugar para descartar o frasco do remédio. (F12)

\section{Classe 5 - Preocupação quanto ao tratamento com os quimioterápicos orais}

Nesta classe, os familiares relataram suas preocupações quanto ao tratamento com quimioterápicos orais, sendo esses aspectos importantes necessidades de aprendizagem a serem abordadas por profissionais envolvidos no cuidado de crianças e adolescentes em quimioterapia oral. As palavras que obtiveram maior frequência de associação foram: imunidade; muito; abaixar; dúvida; quimioterapia. Os familiares mostraram-se preocupados em relação aos efeitos adversos e à queda da imunidade.

Uma dúvida que eu tenho muito grande é se o efeito da quimioterapia acaba atingindo outros órgãos, porque ela já saiu do hospital com a imunidade muito baixa. Essa éa minha dúvida, o quimioterápico faz bem ou faz mal? (F13)

Gostaria de saber o que pode ocasionar porque não me explicaram muito se podia dar alguma reação, eu que procurei saber com um aqui outro ali, tenho dúvidas quanto aos efeitos colaterais. (F7)

O familiar F20 mostrou-se preocupado sobre em quais circunstâncias deve encaminhar a criança para a emergência e qual o procedimento correto caso a criança tenha vomitado após tomar o quimioterápico.

Eu tenho algumas dúvidas: em que situação é importante trazer, imediatamente, para o hospital de referência? Com febre, traz para o hospital ou não? Se ele tomou o quimioterápico e vomitou, em quanto tempo ele é absorvido? Devo dar outro?(F2O)

Nos depoimentos, chamou a atenção que dois familiares relataram desconhecer que a medicação oral que administravam em casa era um quimioterápico. Um familiar, inclusive, mostrou que, na tentativa de obter mais informação para nortear seu cuidado, lê a bula do medicamento, mas que, ainda assim, não tem certeza se está fazendo o cuidado certo.

Eu não sabia que a Mercaptopurina era um quimioterápico, eu achava que era tipo Bactrim, para mim, o quimioterápico era só o MTX. (F21)

Eu tenho uma dúvida sim porque, embora eu não soubesse que esse remédio era quimioterápico, eu li a bula e vi que ele era um remédio bem importante. Minha dúvida é se estou fazendo certo, se realmente é importante ele tomar antes de dormir. (F17)

\section{DISCUSSÃO}

O corpus textual obteve, na análise hierárquica descendente, um bom aproveitamento, com percentagem superior a $75 \% .{ }^{17} \mathrm{Assim}$, o material textual foi considerado útil para realizar, de forma adequada, esse tipo de análise.

Os dados desta pesquisa mostraram que os familiares realizam o cuidado com base nas próprias experiências, a partir de um corpo de conhecimento construído na trajetória de suas vidas, e que nem sempre há diálogos contextualizados com os profissionais que atendem a criança ou o adolescente. Assim, ainda há dúvidas recorrentes acerca, por exemplo, da administração e do manuseio que podem, ao longo do tempo, afetar a condução do tratamento, do armazenamento e do descarte, dentre outros, o que revela necessidades de aprendizagem importantes a serem problematizadas pelos profissionais nas práticas educativas juntos aos familiares. Neste sentido, de acordo com a concepção freiriana, é preciso estabelecer, junto a essas famílias, um processo educativo problematizador baseado no diálogo verdadeiro entre todos os envolvidos, no qual há espaço para ouvir o sujeito e a realidade que vivencia, rompendo, assim, com a verticalidade das práticas bancárias. ${ }^{11}$

Durante o tratamento com os quimioterápicos orais, muitos fatores podem dificultar a adesão à terapêutica e tornar-se uma barreira para o cumprimento das recomendações da equipe multiprofissional, abrangendo a deficiência de entendimento relacionado ao próprio tratamento, complexidade no regime de doses, administração de outros medicamentos, interação com outro medicamento e com alimento, o gasto com a terapia e os efeitos adversos.$^{22}$ Ressalta-se, ainda, que, para a administração de cada quimioterápico, devem ser consideradas suas especificidades quanto ao manejo com alimentos, observando e respeitando os intervalos, atentando também para condutas relacionadas a vômitos pós-administração da droga, considerando o tempo de absorção do medicamento. Reforça-se que, diante dessa gama de necessidades de aprendizagem, é fundamental o estabelecimento de encontros dialógicos ${ }^{11}$ mediante a escuta atenta que facilite o compartilhamento de saberes junto a cada família, de acordo com o antineoplásico oral prescrito, agregando, ao diálogo, suas experiências e conhecimentos prévios.

Nessa diretiva, a dialogicidade precisa ser o fio condutor das práticas educativas em saúde junto a essas famílias, para ser possível conhecer e refletir sobre sua realidade concreta, possibilitando, desse modo, que profissionais e familiares encontrem, juntos, soluções viáveis que facilitem o cuidado no cotidiano da vida. Uma estratégia que pode ser recomendada, aos familiares de crianças e adolescentes, é a utilização de um calendário com o registro de datas, horários e doses dos medicamentos, com um espaço para a checagem de cada ingestão. Esse calendário deve ser avaliado, periodicamente, pela equipe multiprofissional para a avaliação da aderência ao tratamento de cada família, considerando suas fragilidades e potencialidades. ${ }^{23}$

Além disso, as ações de Enfermagem devem englobar orientações quanto à prescrição do quimioterápico, retirando 
as dúvidas quanto à dose, ao horário, à duração do tratamento, aos efeitos adversos, além de estimular o familiar no momento da administração a conversar com a criança e o adolescente, usando estratégias para que seja um momento agradável. ${ }^{23,24}$

Nas entrevistas, os familiares relataram diferentes modos de armazenamento dos quimioterápicos orais, o que indica que há desconhecimento, dúvidas e erros e, portanto, é necessário existir uma abordagem desse tema nas orientações a serem desenvolvidas pelos profissionais de saúde, incluindo o enfermeiro, de maneira participativa, com o intuito de minimizar as complicações de um tratamento inadequado.

Cabe destacar que as condições de armazenamento são importantes para a garantia da eficácia do fármaco, pois o mau acondicionamento pode propiciar a degradação e, com isso, os quimioterápicos podem não fazer o efeito terapêutico desejado. Os quimioterápicos, quando armazenados em locais quentes e úmidos, como cozinha, banheiro ou em ambientes com alta luminosidade, podem sofrer alterações em sua composição, podendo ser químicas, físicas ou microbiológicas, diminuindo a efetividade terapêutica ou elevando o risco de efeitos tóxicos de acordo com o tipo de degradação sofrida pelo fármaco. ${ }^{25}$ Ouvir os sujeitos para entender sua realidade e socializar os saberes ${ }^{11}$ é fundamental para a transformação dos hábitos e a adoção dos cuidados necessários e seguros com os medicamentos antineoplásicos orais.

Alguns familiares relataram que retiram os comprimidos da embalagem original, cortam e os colocam em vidros. Esse cuidado é pautado na própria experiência de vida, como tentativa de buscar solução, demonstrando consciência ingênua sobre essa prática cuidativa. Sobre isso, estudo destacou que a permanência do medicamento na embalagem original é importante, pois ajuda a evitar trocas medicamentosas, quando íntegras e identificadas, além de manter sua estabilidade. ${ }^{25}$ Assim, orientações sobre o adequado condicionamento dos quimioterápicos orais devem ser realizadas durante o tratamento, tendo em vista que essa necessidade de aprendizagem foi evidente nos depoimentos. Desse modo, afastam-se práticas que possam prejudicar a terapêutica das crianças e adolescentes, o que facilitará, aos familiares, transitar da consciência ingênua para a consciência crítica. $^{11}$

Quanto ao manuseio dos quimioterápicos pelos familiares no ambiente domiciliar, evidências indicam que a terapêutica antineoplásica oral necessita conservar as mesmas precauções de segurança que são utilizadas para as terapêuticas endovenosas de maneira que proteja os pacientes, os cuidadores e o ambiente. ${ }^{26}$ Portanto, são necessárias ações educativas para orientações quanto ao manuseio seguro e à adequada administração dos quimioterápicos orais, evitando a colocação direta das mãos nos comprimidos ou cápsulas. Essa manipulação deve ser feita com luvas ou reduzir, ao mínimo, o toque utilizando copo, a própria tampa do frasco ou papel de cozinha, lavando as mãos após o procedimento. Não é indicado partir, abrir, esmagar ou mastigar o quimioterápico a fim de se evitar inalar ou tocar o conteúdo. Aconselha-se também evitar o contato direto com fezes, urina ou outro tipo de excreção e efetuar duas descargas de autoclismo depois da eliminação urinária, fecal ou de outra excreção durante a terapêutica antineoplásica oral e nas $48 \mathrm{~h}$ após o término do tratamento. ${ }^{26,27}$

Além disso, quanto ao descarte dos quimioterápicos, mencionado nas entrevistas, tendo em vista os riscos ambientais que podem ser minimizados, é preciso que se invista em práticas educativas ${ }^{11}$ dialógicas, pautadas no contexto de vida das pessoas e na reflexão crítica para que o conhecimento seja construído. Sabe-se que, com relação ao adequado descarte do lixo decorrente da utilização de agentes citotóxicos, este deve ser acondicionado em recipiente padronizado, fechado e de consistência rígida, que impeça perfuração ou vazamento, para que seja neutralizado ou incinerado. Esses cuidados são importantes para a segurança das pessoas e do ambiente, pois o familiar deve saber o correto destino de medicamentos vencidos, assim como a devolução do quimioterápico não usado para o hospital de referência, para que ocorra a eliminação apropriada. É necessário advertir, ainda, que o quimioterápico não deve ser eliminado por meio da rede de esgotos, pois estes resíduos podem contaminar o meio ambiente. ${ }^{25}$

Os apontamentos dos familiares quanto aos assuntos que gostariam de ter orientações e que geram preocupações também são importantes necessidades de aprendizagem a serem abordadas em práticas educativas. Entre esses assuntos, houve destaque para os efeitos adversos dos medicamentos, sinais e sintomas indicativos da necessidade de retornar ao hospital, armazenamento e manuseio dos medicamentos. Assim, ficaram explícitas lacunas de práticas educativas sobre o tratamento com quimioterápicos orais.

Nessa diretiva, pacientes participantes de um estudo identificaram a importância de existirem informações escritas que pudessem consultar no domicílio e calendários de medicação para realizarem as anotações como um diário. Solicitaram ainda que esses materiais educativos abordassem as seguintes temáticas: informações sobre os efeitos adversos; como e quando tomar o quimioterápico; como a droga funciona; interações medicamentosas alarmes; armazenamento; manuseio; descarte e educação sobre drogas antieméticas. ${ }^{23}$ Tais resultados assemelham-se com os desta pesquisa e reforçam a necessidade de estudos e ações nesse contexto.

A educação em saúde sobre medicamentos oncológicos pode ser complexa, pois exige que o enfermeiro oriente os familiares quanto aos cuidados específicos com os antineoplásicos orais, abordando questões importantes sobre a higiene e alimentação; manipulação dos quimioterápicos; dosagem correta dos medicamentos; fatores de risco que podem levar à neutropenia febril e infecções; efeitos adversos relacionados ao tratamento; quando os familiares devem encaminhar as crianças ou os adolescentes à emergência e orientação sobre os medicamentos usados para prevenir e tratar os sintomas, como, por exemplo, antieméticos, anti-histamínicos, corticosteroides e analgésicos, dentre os quais muitos são medicamentos de alto risco e também requerem monitoramento rigoroso do familiar e 
da equipe de saúde. ${ }^{28,29}$ Logo, diante da complexidade desse tipo de cuidado, os enfermeiros precisam realizar práticas educativas que superem a mera narrativa e que proporcionem condições para uma tomada de consciência e atitude crítica no sentido de haver mudanças reais da realidade, de maneira voluntária e autônoma, por parte dos familiares.

Ao partir do pressuposto de que o enfermeiro tem papel fundamental no manejo do regime terapêutico desta clientela, recomenda-se a sua participação efetiva no processo educativo em saúde na transição da administração dos quimioterápicos orais para o contexto domiciliar e seu posterior acompanhamento, buscando desenvolver a capacidade de autogerenciamento dos familiares, favorecendo a reflexão crítica sobre sua situação e ações, para garantir a segurança da criança ou do adolescente e reduzir, ao mínimo possível, os riscos e danos desnecessários associados ao cuidado em saúde e à adesão e eficácia do tratamento. ${ }^{30}$

Como limitação deste estudo, é possível destacar que os resultados refletem, exclusivamente, a vivência de uma instituição, o que pode não exprimir uma totalidade de pessoas que experienciam este universo.

\section{CONCLUSÃO E IMPLICAÇÕES PARA A PRÁTICA}

Os achados do estudo permitiram descrever as necessidades de aprendizagem dos familiares frente ao tratamento domiciliar junto às crianças e aos adolescentes em tratamento com quimioterápicos antineoplásicos orais, além de desvelar o modo como a terapêutica antineoplásica é realizada por eles. Notase que, por vezes, muitos cuidados são executados de forma equivocada, contrapondo-se às recomendações da literatura científica, o que pode causar riscos para as pessoas e prejudicar o ambiente onde estão inseridas.

As necessidades de aprendizagem referidas pelos familiares, sendo estas, pontos-chave para ações de educação em saúde, foram relacionadas à forma adequada de administração, armazenamento e manipulação dos quimioterápicos orais. Eles também gostariam de ter orientações quanto aos efeitos colaterais e aos sinais e sintomas que indicam a necessidades de levar a criança e o adolescente à emergência do hospital. Tais temáticas precisam ser problematizadas no desenvolvimento de práticas educativas dialógicas por parte dos profissionais de saúde, incluindo o enfermeiro.

Com relação às implicações para a prática, ressalta-se a importância da equipe de Enfermagem em prover, aos familiares, subsídios para sanar dúvidas e dificuldades decorrentes da terapêutica para que o tratamento quimioterápico seja realizado de forma adequada e que a família se sinta apta para cuidar da criança e do adolescente, garantindo, assim, que o uso do medicamento seja administrado de forma segura e eficaz.

Este estudo enriquece o conhecimento existente sobre a temática, pois propicia um aprofundamento teórico e promove a reflexão sobre o câncer infantojuvenil de forma que é capaz de incentivar e sensibilizar os profissionais da saúde sobre a importância das práticas educativas no tratamento com antineoplásicos orais.

\section{CONTRIBUIÇÕES DOS AUTORES}

Desenho do estudo. Gabriele Alvernaz Silva Franco. Liliane Faria da Silva.

Coleta ou produção dos dados. Gabriele Alvernaz Silva Franco.

Análise de dados. Gabriele Alvernaz Silva Franco. Liliane Faria da Silva. Flavio Luiz Seixas. Fernanda Garcia Bezerra Góes. Ana Carolina Alves Vollu. Eduardo Charpinel Lagoeiro.

Interpretação dos resultados. Gabriele Alvernaz Silva Franco.

Liliane Faria da Silva. Fernanda Garcia Bezerra Góes.

Redação e revisão crítica do manuscrito. Gabriele Alvernaz Silva Franco. Liliane Faria da Silva. Flavio Luiz Seixas. Fernanda Garcia Bezerra Góes. Ana Carolina Alves Vollu. Eduardo Charpinel Lagoeiro.

Aprovação da versão final do artigo. Gabriele Alvernaz Silva Franco. Liliane Faria da Silva. Flavio Luiz Seixas. Fernanda Garcia Bezerra Góes. Ana Carolina Alves Vollu. Eduardo Charpinel Lagoeiro.

Responsabilidade por todos os aspectos do conteúdo e a integridade do artigo publicado. Gabriele Alvernaz Silva Franco. Liliane Faria da Silva. Flavio Luiz Seixas. Fernanda Garcia Bezerra Góes. Ana Carolina Alves Vollu. Eduardo Charpinel Lagoeiro.

\section{EDITOR ASSOCIADO}

Aline Cristiane Cavachilli Okido (D)

\section{EDITOR CIENTÍFICO}

Ivone Evangelista Cabral (D)

\section{REFERÊNCIAS}

1. Instituto Nacional do Câncer José Alencar Gomes da Silva. Estimativa 2020: incidência de câncer no Brasil [Internet]. Rio de Janeiro: INCA 2020 [citado 2021 ago 26]. Disponível em: https://www.inca.gov.br/ publicacoes/livros/estimativa-2020-incidencia-de-cancer-no-brasil

2. Alves EA, Tavares GG, Borges LL. Importância da atenção farmacêutica para a quimioterapia antitumoral. Revista Brasileira Militar de Ciências. 2020;6(15):8-17. http://dx.doi.org/10.36414/rbmc.v6i15.35.

3. De Mesquita MER, Da Silva RP. Autocuidado e quimioterapia ora domiciliar: avaliação das práticas educativas dos enfermeiros sob a perspectiva de pacientes. Rev Bras Cancerol. 2016;62(3):237-45 http://dx.doi.org/10.32635/2176-9745.RBC.2016v62n3.165.

4. Kinnaer L-M, De Coster S, Coolbrandt A, Decoene E, Van Hecke A, Foulon V. Key elements for the education and counselling of patients treated with oral anticancer drugs. Eur J Oncol Nurs. 2019;41:173-94 http://dx.doi.org/10.1016/j.ejon.2019.06.010. PMid:31358251.

5. Rudnitzki T, McMahon D. Oral agents for cancer: Safety challenges and recommendations. Clin J Oncol Nurs. 2015;19(3, Suppl):41-6. http:// dx.doi.org/10.1188/15.S1.CJON.41-46. PMid:26030392.

6. Held K, Ryan R, Champion JM, August K, Radhi MA. Caregiver survey results related to handling of oral chemotherapy for pediatric patients with acute lymphoblastic leukemia. J Pediatr Hematol Oncol. 2013;35(6):e249-53. http://dx.doi.org/10.1097/MPH.0b013e31827e4a73. PMid:23274379. 
7. Silva LN, Silva LF, Goes FGB, Machado MED, Paiva ED. Orientações sobre quimioterapia junto à criança com câncer: método criativo sensível. Online Braz J Nurs [Internet] 2015 [citado 2020 dez 29]; 14(4):471-80. Disponível em: http://www. objnursing.uff.br/index.php/nursing/article/ view/5310

8. Lopes VJ, Shmeil MAH. Evaluation of computer-generated guidelines for companions of paediatric patients undergoing chemotherapy. Rev Gaúcha Enferm. 2016; 37(Spec No):e67407. http://dx.doi.org/10.1590/19831447.2016.esp.67407.

9. Sueiro IM, Góes FGB, Silva LF, Moraes JRMM. Nursing care towards feeding children undergoing chemotherapy treatment: Collière's contributions. Rev Fund Care Online. 2019;11(Spec No):351-7. http:// dx.doi.org/10.9789/2175-5361.2019.v11i2.351-357.

10. Rodrigues JRG, Siqueira Jr AC, Siqueira FPC. Nursing consultation in pediatric oncology: a tool for empowering parents. Rev Fund Care Online. 2020;12:210-20. http://dx.doi.org/10.9789/2175-5361.rpcfo. v12.7569.

11. Freire P. Pedagogia da Autonomia: saberes necessários à prática educativa. 43a ed. São Paulo: Paz e Terra; 2011.

12. Pitano SC. A educação problematizadora de Paulo Freire, uma pedagogia do sujeito social. Interação (Curitiba). 2017;42(1):87-104. http://dx.doi. org/10.5216/ia.v42i1.43774.

13. Precce ML, Moraes JRMM. Educative process with relatives of children with special health needs in the hospital-home transition. Texto Contexto Enferm. 2020;29:e20190075. http://dx.doi.org/10.1590/1980-265xtce-2019-0075.

14. Taquette SR, Minayo MCS. Análise de estudos qualitativos conduzidos por médicos publicados em periódicos científicos brasileiros entre 2004 e 2013. Physis. 2016;26(2):417-34. http://dx.doi.org/10.1590/ S0103-73312016000200005.

15. Tong A, Sainsbury P, Craig J. Consolidated criteria for reporting qualitative research (COREQ): a 32-item checklist for interviews and focus groups. Int J Qual Health Care. 2007;19(6):349-57. http://dx.doi.org/10.1093/ intghc/mzm042. PMid:17872937.

16. Saunders B, Sim J, Kingstone T, Baker S, Waterfield J, Bartlam B et al Saturation in qualitative research: exploring its conceptualization and operationalization. Qual Quant. 2018;52(4):1893-907. http://dx.doi. org/10.1007/s11135-017-0574-8. PMid:29937585.

17. Souza MAR, Wall ML, Thuler ACMC, Lowen IMV, Peres AM. The use of IRAMUTEQ software for data analysis in qualitative research. Rev Esc Enferm USP. 2018;52:e03353. http://dx.doi.org/10.1590/s1980220x2017015003353. PMid:30304198.

18. Góes FGB, Santos AST, Campos BL, Silva ACSS, Silva LF, França LCM. Utilização do software IRAMUTEQ em pesquisa de abordagem qualitativa: relato de experiência. Rev Enferm UFSM. 2021;11(e63):122. http://dx.doi.org/10.5902/2179769264425.

19. Almico T, Faro A. Enfrentamento de cuidadores de crianças com câncer em processo de quimioterapia. Psicol Saude Doencas. 2014;15(3):72337. http://dx.doi.org/10.15309/14psd150313.
20. Corrêa VB, Silva LF, Goes FGB, Nunes MDR, Pacheco STA, Silveira ALD. Temas para práticas educativas sobre cuidados domiciliares às crianças com leucemia em uso de cateter venoso central semi-implantável. Rev Min Enferm. 2020;24:e-1347. http://dx.doi. org/10.5935/1415.2762.20200084.

21. Ministério da Saúde (BR). Comissão Nacional de Ética em Pesquisa Orientações para condução de pesquisas e atividade dos cep durante a pandemia provocada pelo coronavírus Sars-cov-2 (COVID-19) [Internet]. Brasília: Ministério da Saúde; 2020 [citado 2020 dez 29]. Comunicado CONEP. Disponível em: http://www.fo.usp.br/wpcontent/ uploads/2020/07/Orienta\%C3\%A7\%C3\%B5escondu\%C3\%A7\%C3\% A3o-de-pesquisas-e-atividades-CEP.pdf

22. Linder LA, Wu YP, Macpherson CF, Fowler B, Wilson A, Jo Y et al. Ora medication adherence among adolescents and young adults with cancer before and following use of a smartphone-based medication reminder app. J Adolesc Young Adult Oncol. 2019;8(2):122-30. http:// dx.doi.org/10.1089/jayao.2018.0072. PMid:30307778.

23. Lambourne T, Minard LV, Deal H, Pitman J, Rolle M, Saulnier D et al Optimizing patient education of oncology medications: a patient perspective. J Cancer Educ. 2019;34(5):1024-30. http://dx.doi.org/10.1007/s13187018-1406-9. PMid:30074227.

24. Shinnick S. Assessment of the role of the pediatric nurse in patient education and follow-up of patients receiving oral anticancer treatment. J Pediatr Oncol Nurs. 2020;37(1):46-54. http://dx.doi. org/10.1177/1043454219871081. PMid:31475636.

25. Pereira MBL, Paula MCA, Santos NL. Medicamentos: descarte de vencidos e utilização de sobras. Rev Saúde (St. Maria). 2018;44(3):1-8. https://doi.org/10.5902/2236583425355.

26. Ribed A, Escudero-Vilaplana V, Romero-Jimenez RM, IglesiasPeinado I, Herranz-Alonso A, Sanjurjo-Saez M. Guiding pharmacist clinical interviews: a safety tool to support the education of patients treated with oral antineoplastic agents. Expert Opin Drug Saf. 2016;15(4):427-35. http://dx.doi.org/10.1517/14740338.2016.115 0998. PMid:26854363.

27. Instituto Nacional do Câncer José Alencar Gomes da Silva Quimioterapia: orientações aos pacientes [Internet]. Rio de Janeiro: INCA; 2018 [citado 2021 nov 20]. Disponível em: https://www.inca.gov. $\mathrm{br} /$ publicacoes/cartilhas/quimioterapia

28. Marshall VK, Cairns PL. Challenges of caregivers of cancer patients who are on oral oncolytic therapy. Semin Oncol Nurs. 2019;35(4):363-9. http://dx.doi.org/10.1016/j.soncn.2019.06.009. PMid:31229341.

29. Lively A, Minard LV, Scott S, Deal H, Lambourne T, Giffin J. Exploring the perspectives of healthcare professionals in delivering optimal oncology medication education. PLoS One. 2020;15(2):e0228571. http://dx.doi. org/10.1371/journal.pone.0228571. PMid:32049970.

30. Okido ACC, Cunha ST, Neves ET, Dupas G, Lima RAG. Technologydependent children and the demand for pharmaceutical care. Rev Bras Enferm. 2016;69(4):718-24. http://dx.doi.org/10.1590/00347167.2016690415i. PMid:27508478. 